\title{
Indépendance au Brésil et Lumières au Portugal : politique et culture dans l'espace luso-brésilien (1792-1823)
}

Independence in Brazil and Enlightenment in Portugal: Politics and Culture in the Portuguese-Brazilian world (1792-1823)

Lúcia Maria Bastos Pereira Das Neves et Guilherme Pereira Das Neves Traducteur : Diego Fonseca dos Santos

\section{(2) OpenEdition} Journals

Édition électronique

URL : https://journals.openedition.org/ahrf/12103

DOI : $10.4000 / a h r f .12103$

ISSN : 1952-403X

Éditeur :

Armand Colin, Société des études robespierristes

Édition imprimée

Date de publication : 1 septembre 2011

Pagination : $31-53$

ISBN : 978-2-200-92700-4

ISSN : 0003-4436

Référence électronique

Lúcia Maria Bastos Pereira Das Neves et Guilherme Pereira Das Neves, «Indépendance au Brésil et Lumières au Portugal : politique et culture dans l'espace luso-brésilien (1792-1823) », Annales historiques de la Révolution française [En ligne], 365 I Juillet-septembre 2011, mis en ligne le 01 septembre 2014, consulté le 23 avril 2022. URL : http://journals.openedition.org/ahrf/12103; DOI : https://doi.org/10.4000/ahrf.12103 


\title{
INDÉPENDANCE AU BRÉSIL ET LUMIÈRES AU PORTUGAL : POLITIQUE ET CULTURE DANS L'ESPACE LUSO-BRÉSILIEN (1792-1823)
}

\author{
Lúcia Maria BASTOS PEREIRA DAS NEVES \\ Guilherme PEREIRA DAS NEVES
}

\begin{abstract}
Dès 1772 , des natifs de l'Amérique portugaise eurent des contacts avec les Lumières à Coïmbra et occupèrent des postes au Portugal et dans ses possessions d'outre-mer. Si la chute de Pombal en 1777 ne causa pas un vrai changement d'orientation politique, la noblesse traditionnelle reprit assez de pouvoir pour contraindre le souverain. Cependant, ce fut la présence de Rodrigo de Sousa Coutinho au Cabinet du prince régent João qui assura l'action réformatrice la plus inspirée des Lumières. En 1803, l'option diplomatique prise par la couronne portugaise le contraignit à quitter ce poste jusqu'à ce que les pressions de Bonaparte se traduisent par l'invasion du royaume portugais et le départ de la Cour pour Rio de Janeiro. En dépit de ces bouleversements, la politique resta confinée à l'espace privé et la religion demeura la forme dominante de structuration du monde. Dans ces conditions, malgré une importante circulation de pamphlets et de périodiques aux idées libérales, l'Indépendance du Brésil en 1822, conduite par l'héritier du trône lusitanien lui-même, se limita à une dispute avec le Portugal pour l'hégémonie à l'intérieur de l'empire, mais légua néanmoins des tensions profondes au nouveau pays.
\end{abstract}

Mots-clés : Lumières, religion, cour, politique, indépendance.

Comme le remarque l'historien colombien Germán Colmenares, à de rares exceptions près, l'historiographie latino-américaine $\mathrm{du} \mathrm{XIX}^{\mathrm{e}}$ siècle, devant le besoin d'affirmer l'autonomie de nouvelles nations, fut poussée à considérer que l'indépendance par rapport aux anciennes métropoles ibériques avait coupé les racines de la condition coloniale. De ce fait, elle 
légua aux générations suivantes un grave problème lorsque ce passé, que l'on supposait aboli, surgit de nouveau dans les coutumes, dans l'ignorance et dans les préjugés ${ }^{1}$. Outre quelques visionnaires antérieurs, ce n'est qu'à partir de 1930 que les historiens brésiliens commencèrent à s'en rendre compte ${ }^{2}$. Suivant la même perspective, cet article cherche à considérer la politique de l'indépendance du Brésil en 1822 comme liée, d'abord à l'environnement culturel portugais des dernières années du $\mathrm{XVIII}^{\mathrm{e}}$ siècle, ensuite, des deux côtés de l'Atlantique, à la survivance des pratiques caractéristiques de l'Ancien Régime et, finalement, au jeu des renversements politiques qui marquèrent cette époque-là.

\section{Les Lumières et l'empire (1750-1800)}

Bien qu'il soit de plus en plus difficile d'établir, comme il était convenu, une relation univoque entre les Lumières et les actions du marquis de Pombal pendant le gouvernement de José $\mathrm{I}^{\mathrm{er}}$ (1750-1777), quelques mesures prises alors indiquent une conscience croissante de la capacité d'intervention du pouvoir central de transformer et adapter la réalité à ses intérêts propres, et suggèrent aussi combien la couronne portugaise assumait déjà les formes d'un état de police ${ }^{3}$. Les actions que le tremblement de terre de 1755 exigea en constituent la démonstration la plus frappante. Dans une première étape (1755-1761), cela se montre par la formation des compagnies de commerce à caractère mercantiliste, par la soumission de la haute noblesse, par l'expulsion des Jésuites, enfin par la création de l'Intendance de Police et du Trésor Royal. Dans une seconde étape (1768-1774), en plus de l'application de quelques mesures

(1) Germán Colmenares (1938-1990), Las convenciones contra la cultura : ensayos sobre historiografia hispanoamericana del siglo XIX (1967), Medellín, La Carreta, 2008, p. 17-18.

(2) La nouvelle perspective peut être datée à partir de l'œuvre de Gilberto FreYre, Casagrande e senzala (1933, Maîtres et esclaves, 1952) et de celle de Sérgio BuARQue de Holanda, Raízes do Brasil (1936, Racines du Brésil, 1998), mais elle a été amplifiée grâce à la vision de Charles R. BOXER, The Portuguese Seaborne Empire (1969).

(3) Pierangelo SchiERA, «A 'polícia' como síntese de ordem e de bem-estar no moderno Estado centralizado » dans Antônio Manuel Hespanha (dir.), Poder e instituições na Europa do Antigo Regime : colectânea de textos, Lisboa, Fundação Calouste Gulbenkian, 1984, p. 307-19; Marc RaEfF, «The Well-Ordered Police State and the Development of Modernity in Seventeenth - and Eighteenth-Century Europe : An Attempt at a Comparative Approach », The American Historical Review, no 80/5, décembre 1985, p. 1221-43; José Damião RoDRIGUES, "Para o socego e tranqüilidade pública das ilhas : fundamentos, ambição e limites das reformas pombalinas nos Açores », Tempo, n 21, juin 2006, p. 144-70; José Manuel SuBTIL, O terremoto político (1755-1759) - memória e poder, Lisboa, EDIUAL, 2007 ; Nuno Gonçalo Freitas MonTEIro, D. José, na sombra de Pombal, Mem Martins, Círculo de Leitores, 2008. 
législatives importantes, par la création de l'Imprimerie Royale, du Tribunal Royal de la Censure et par la mise en place de la réforme de l'enseignement supérieur, accompagnée par l'achèvement de celle, déjà tardive, des études élémentaires. Entre-temps, les effets de la Guerre de Sept Ans sur l'échiquier diplomatique européen avaient mis en évidence non seulement la situation chancelante du petit royaume péninsulaire devant les puissances qui émergeaient, mais avaient aussi prouvé la fragilité militaire d'un empire qui s'étendait sur quatre continents. Comme résultat, bien que le Portugal eût beau adopter une politique de neutralité, il ne parvint pas à rompre avec la traditionnelle alliance anglaise ${ }^{4}$.

Cette vulnérabilité extérieure ne fut pas modifiée par l'accession au trône de Maria $\mathrm{I}^{\text {re }}$ et la chute de Pombal en 1777; à l'inverse de ce que l'historiographie a longtemps soutenu, il n'y a pas eu de tournant (ou Viradeira) dans la politique de la couronne ${ }^{5}$. De nombreux prisonniers d'un rang social élevé sortirent pourtant de leurs cellules, des émigrés revinrent de l'étranger et la rancœur contre le marquis de Pombal s'exprima par une production fébrile de pamphlets manuscrits qui n'ont pas encore été suffisamment étudiés ${ }^{6}$. Durant les années suivantes, l'intolérance religieuse incita l'Inquisition à étouffer des comportements tenus pour libertins, comme fut le cas lors du procès contre Anastácio da Cunha, professeur de géométrie à Coimbra, ou de celui d'un savetier qui dénonça les propos irrévérencieux qui se tenaient dans une boutique d'apothicaire à Rio de Janeiro ${ }^{7}$. Cependant, la remise sous presse de la Gazette de Lisbonne, interdite depuis 1762, l'apparition de nouveaux périodiques et d'espaces de sociabilité inédits, l'accroissement du marché de livres, le rôle pris par le théâtre auprès des élites et, en 1779, la création de 1'Académie Royale des Sciences de Lisbonne, sur proposition du Duc

(4) Vitorino Magalhães Godinho, A estrutura da antiga sociedade portuguesa, Lisboa, Arcádia, 1971, p. 11-31; Francisco Falcon, A época pombalina, São Paulo, Ática, 1982; JoséAugusto FrançA, Lisboa pombalina e o iluminismo, Lisboa, Bertrand, 1987; Jorge Borges de MACEDo, História diplomática portuguesa : constantes e linhas de força, Lisboa, Tribuna da História, 2008.

(5) Pour un aperçu plus général, voir Guilherme P. NEves, « Do Império luso-brasileiro ao Império do Brasil (1789-1822) », Ler História, n²7-28, 1995, p. 75-103, et Bartolomé BenNASSAR et Richard MARIN, Histoire du Brésil, 1500-2000, Paris, Fayard, 2000.

(6) J. J. CARVAlhão SANTOS, Literatura e política : pombalismo e antipombalismo, Coimbra, Minerva, 1991. Patrícia CARDOSo Lins Alves va soutenir une thèse à l'Universidade Federal Fluminense sur cette question, orientée dans sa recherche au Portugal par Tiago dos Reis Miranda (UNL).

(7) Cf. João Pedro Ferro (éd.), O Processo de José Anastácio da Cunha na Inquisição de Coimbra (1778), Lisboa, Palas, 1987 et David Higgs (éd.), « O Santo Ofício da Inquisição de Lisboa e 'a Luciferina Assembléia' do Rio de Janeiro na década de 1790 », Revista do Instituto Histórico e Geográfico Brasileiro, (dorénavant, RIHGB), n 412, juillet-septembre 2001, p. 239-384. 
de Lafões, favorisèrent la circulation de nouvelles idées ${ }^{8}$. Si l'intendant de police, Pina Manique, figure emblématique de l'époque, s'occupa, d'une part, de l'éclairage public de Lisbonne et de la fondation d'une institution pour l'accueil des orphelins et des pauvres, il faut remarquer d'autre part que, à partir de 1793, il voyait en chaque Français un Jacobin potentiel ${ }^{9}$. En conséquence, l'abbé Correia da Serra, secrétaire de l'Académie, s'exile en 1795 craignant des représailles parce qu'il avait hébergé un naturaliste français Girondin; une décennie plus tard, après être resté deux ans dans les geôles de l'Inquisition sous l'accusation d'être francmaçon ${ }^{10}$, Hipólito José da Costa s'évade pour aller fonder un journal à Londres $^{11}$. De 1751 à 1800 , alors qu'étaient publiés les volumes de la Recreação filosófica du père Teodoro de Almeida sur la compatibilité entre la religion et la connaissance du monde naturel, Antônio Cândido de Figueiredo, prêtre lui aussi, adaptait, après l'expulsion des Jésuites, les conceptions du fébronianisme au Portugal et on refusait alors à Ludovico Antonio Muratori, en s'inspirant de l'Encyclopédie elle-même, la condition d'auteur de Il cristianesimo felice (1743), une des œuvres qui inspira l'idée d'utopie, selon Girolamo Imbruglia ${ }^{12}$. Si frère Manuel do Cenáculo, président du Tribunal Royal de la Censure, et qui avait participé à la réforme universitaire, tomba en défaveur, il continuait néanmoins à être lié aux activités culturelles du royaume pendant plus de trente ans. Pour sa part, le prêtre Antônio Ribeiro dos Santos, professeur à Coimbra pendant le ministère de Pombal, attaqua les principes absolutistes que le juriste

(8) André Belo, As gazetas e os livros : a Gazeta de Lisboa e a vulgarização do impresso (1715-1760), Lisboa, ICS, 2001 et Diogo RAMADA CURTo et al., As gentes do livro. Lisboa, século XVIII, Lisboa, Biblioteca Nacional, 2007.

(9) Adérito Tavares et José dos Santos Pinto, Pina Manique : um homem entre duas épocas, Lisboa, Casa Pia de Lisboa, 1990.

(10) Il avait été emprisonné alors qu'il revenait d'un voyage à l'extérieur effectué sur ordre de la Couronne,

(11) Établi à Londres, il lança (1808) le Correio Braziliense, le plus important périodique de l'époque. Cf. Sérgio Góes de Paula (dir.), Hipólito José da Costa, São Paulo, Ed. 34, 2001, p. 13-36; Alberto Dines (ed.), Hipólito José da Costa e o Corrreio Braziliense. Estudos, São Paulo / Brasília, Imprensa Oficial / Correio Braziliense, 2002.

(12) L'invenzione del Paraguay: studio sull'idea di comunità tra Seicento e Settecento, Napoli, Bibliopolis, 1983. Cf. José de Seabra Da Silva, Dedução cronológica e analítica, Lisboa, Francisco Borges de Sousa, 1767, v. 1, p. 394; Francisco Contente Domingues, « Um projeto enciclopédico e pedagógico : a Recreação Filosófica de Teodoro de Almeida », Revista de História das Idéias, v. 10, 1988, p. 235-48 et Evergton SALES SouZA, Jansénisme et réforme de l'Église dans l'empire portugais, 1640 à 1790, Paris, Centre Culturel Calouste Gulbenkian, 2004. 
Pascoal de Melo Freire essaya d'instiller dans un nouveau code de lois ${ }^{13}$. À la fois poète et érudit, il fréquenta les salons aristocratiques, dirigea la Bibliothèque publique créée en 1796 et critiqua férocement l'évêque Azeredo Coutinho, son collègue à l'Académie, qui, avec un esprit « brutalement pragmatique », n'hésita pas à défendre le trafic d'esclaves pour combattre les doctrines impies de «nouveaux philosophes » français ${ }^{14}$. En 1794, le scandale déclenché par la publication de l'œuvre Medicina teológica de Francisco de Melo Franco, né en Amérique et ayant fait ses études à Coimbra, cause le retour de la censure sur le modèle de l'ancien système tripartite d'avant 1768 : le roi, l'Église et l'Inquisition ${ }^{15}$.

L'ambiance qui règne alors comporte plusieurs des caractéristiques des Lumières, si l'on pense à la sociabilité, à la sensibilité et même à quelques mesures éclairées prises par l'administration. Pourtant, les initiatives semblent toutes revenir au clergé, et le patronage à la Couronne, à l'Église ou à la noblesse. Les discours se maintiennent dans l'univers des vérités de la foi, hésitant à dépasser des propositions pratiques ponctuelles, comme le révèlent la plupart des mémoires économiques de l'Académie des Sciences ${ }^{16}$. La personnalité de Rodrigo de Sousa Coutinho s'inscrit contre cette toile de fond ${ }^{17}$. Filleul de Pombal, il avait reçu une éducation recherchée et fréquenté le cercle d'Anastácio da Cunha, dont il s'éloigne, en 1778, pour effectuer un voyage d'initiation politique [le Wanderjahr], qui l'emmène à Madrid, à Paris, en Suisse et à Turin, où il occupera plusieurs années durant le poste de représentant portugais. À ce « carrefour culturel de l'Europe », il lit Adam Smith et

(13) Jacques Marcadé, Frei Manuel do Cenáculo Vilas Boas, évêque de Beja, archevêque d'Evora (1770-1814), Paris, Centro Cultural Português / Fundação Calouste Gulbenkian, 1978 et José Esteves Pereira, O pensamento político em Portugal no século XVIII : António Ribeiro dos Santos, Lisboa, Imprensa Nacional / Casa da Moeda, 1983.

(14) Guilherme P. Neves, « Guardar mais silêncio do que falar : Azeredo Coutinho, Ribeiro dos Santos e a escravidão ", dans José Luís CARDoso (dir.), A economia política e os dilemas do império luso-brasileiro (1790-1822), Lisboa, CNCDP, 2001, p. 13-62.

(15) Cf. Lúcia Bastos P. Neves, «Um silêncio perverso : censura, repressão e o esboço de uma primeira esfera pública de poder (1820-1823) », dans Maria Luiza TuCCI CARNeIRo (dir.), Minorias silenciadas : história da censura no Brasil, São Paulo, EdUSP / Imprensa Oficial do Estado / Fapesp, 2002, p. 121-53.

(16) Guilherme P. Neves, « Como um fio de Ariadne no intrincado labirinto do mundo : a idéia do império luso-brasileiro em Pernambuco (1800-1822) », Ler História, Lisboa, v. 39, p. 35-58, 2000 et José Luís CARdoso (dir.), Memórias económicas da Academia Real das Ciências de Lisboa... (1789-1815), Lisboa, Banco de Portugal, 1990-1991, 5v.

(17) Andrée Mansuy-Diniz Silva, Portrait d'un homme d'État: D. Rodrigo de Souza Coutinho, Comte de Linhares, 1755-1812, Lisboa/ Paris, CNCDP / Centre Culturel Calouste Gulbenkian, 2002-2006, 2v. 
réfléchit aux réformes adoptées par le royaume sarde; il suit le déroulement des événements européens au cours des années 1780 et regarde de façon critique l'arrivée des premiers réfugiés de la Révolution ${ }^{18}$. Rappelé à Lisbonne en 1796, il y assume le portefeuille de la Marine et des Domaines d'Outre-mer, où il devient un élément moteur de la création de projets dont l'objectif majeur est d'assurer la survie du pouvoir royal au Portugal, dans un contexte chaque fois plus hostile. Pour atteindre ce but, il compte sur le potentiel des territoires d'outre-mer et surtout de l'Amérique. Ainsi, dans la droite ligne des idées de Pombal, il se propose d'intervenir sur la réalité afin de la transformer en ayant recours à des plans, à des mémoires, à des informations nouvelles et à quelques initiatives qu'il mène à bon terme. Grâce à l'esprit plus éclairé qui régnait à l'époque de Maria I ${ }^{\text {re }}$, il se soucie aussi de soulager les pires asservissements dont la population faisait l'objet, de rationaliser l'administration et la justice, de dynamiser la vie économique et d'assurer ainsi que « le Portugais né aux quatre coins du monde se considère uniquement Portugais et ne se souvienne que de la gloire et de la grandeur de la monarchie à laquelle la fortune lui a fait appartenir $»^{19}$.

Les propositions de Sousa Coutinho rencontrèrent un écho. Au Pernambouc, le naturaliste Manuel de Arruda Câmara donne son nom à une variété de plante; à Bahia, le professeur de grec Luís dos Santos Vilhena le surnomme "Patriphile" quand il lui écrit la dédicace de quatre dernières lettres de son œuvre; à Rio de Janeiro, le poète Manuel Inácio da Silva Alvarenga le remercie de l'aide qu'il lui a apportée pour le sortir d'un emprisonnement causé par un abus d'autorité du vice-roi, et lui fait savoir qu'il se reconnaît comme sa créature ${ }^{20}$. À Lisbonne, afin d'imprimer des œuvres capables d'éclairer les agents économiques de l'empire, D. Rodrigo créa la typographie de l'Arco do $\operatorname{Cego}^{21}$, qui réunissait auteurs, correcteurs et traducteurs sous la tutelle du frère Conceição Veloso, naturaliste, cousin de Tiradentes, ce militaire pendu en 1792 pour avoir participé à la révolte connue sous le nom d'Inconfidência de Minas

(18) Andrée Mansuy-Diniz Silva, «L'année 1789 vue de Turin par un diplomate portugais », Dix-Huitième Siècle, n 20, 1988, p. 289-313. Pour la citation, cf. Robert MANDRou, L'Europe absolutiste : raison et raison d'état (1649-1775), Paris, Arthème Fayard, 1977, p. 284.

(19) Rodrigo de Sousa Coutinho, Textos políticos, económicos e financeiros, 1783-1811, Andrée Mansuy-Diniz Silva, (éd.), Lisboa, Banco de Portugal, 1993, v. 2, p. 49.

(20) Cf. Guilherme P. Neves, «A suposta conspiração de 1801 em Pernambuco : idéias ilustradas ou conflitos tradicionais? », Revista Portuguesa de História, n 33, 1999, p. 439-481.

(21) L'arc de l'aveugle. 
Gerais $^{22}$. Plusieurs d'entre eux, ainsi que d'autres personnages placés à des postes-clé dans le royaume et dans l'outre-mer, appartenaient au groupe de presque cinq cents personnes nées en Amérique qui avaient fait leurs études entre 1772 et 1800 à l'Université de Coimbra réformée. Cette petite élite, signalée par Varnhagen dès la deuxième moitié du XIX ${ }^{\mathrm{e}}$ siècle et étudiée par Maria Odila da Silva Dias à la fin des années 1960, fut appelée par Kenneth Maxwell la "Génération de 1790" de laquelle il ne faut pas exclure ceux qui étaient nés en métropole et partageaient les mêmes idées $^{23}$.

\section{La politique et la Cour (1792-1807)}

«Aliénée par les spectres de la Révolution Française », Maria I ${ }^{\text {re }}$ quitte le gouvernement en $1792^{24}$. À la suite de la mort du dauphin (1788), c'est João, son deuxième fils, qui assume la régence. Il a alors 25 ans et, jusqu'à sa mort, en 1826, il sera à la fois exalté et dénigré ${ }^{25}$. Toutefois, dans une conjoncture tumultueuse, il eut l'habileté ou la fortune nécessaire pour se maintenir sur le trône, en dépit des graves problèmes qu'il dut affronter.

Le Portugal, dépourvu d'une armée moderne et ne comptant que 2,5 millions d'habitants, dut observer une position de neutralité apparente

(22) Fernanda Maria Guedes de CAmpos et al., A Casa Literária do Arco do Cego (17991801) : bicentenário, Lisboa, Biblioteca Nacional / Imprensa Nacional / Casa da Moeda, 1999. En ce qui concerne Tiradentes, il a été élevé au rang mythique de fondateur de la nation, après la proclamation de la république, soit un siècle plus tard, cf. José Murilo de Carvalho, A formação das almas, São Paulo, Companhia das Letras, 1990.

(23) Cf. Maria Odila DA Silva Dias, «Aspectos da ilustração no Brasil », RIHGB, n 278, jan.-mar. 1968, p. 105-70; Kenneth MAXwell, «A geração de 1790 e a idéia do império lusobrasileiro » dans Chocolate, piratas e outros malandros, São Paulo, Paz e Terra, 1999, p. 157207; Francisco Adolfo de Varnhagen, História Geral do Brasil (1854-1857), $3^{\text {a }}$ ed., São Paulo, Companhia Melhoramentos (s/d), v. 5, p. 8-19; Ana Cristina Araújo (dir.), O marquês de Pombal e a Universidade, Coimbra, Imprensa da Universidade, 2000; Francisco de MoraIs, « Estudantes da Universidade de Coimbra nascidos no Brasil », Brasília, no 4 (suplemento), 1949, p. 1-599.

(24) Citation de Raymundo Faoro, Os donos do poder, Porto Alegre / São Paulo, Globo / EdUSP, 1975, p. 748. Voir Caetano BeIRÃo, D. Maria I, 1777-1792, Lisboa, Empresa Nacional de Publicidade, 1944 et Luís de Oliveira Ramos, D. Maria I, Mem Martins, Círculo de Leitores, 2007.

(25) Manuel de Oliveira Lima, D. João VI no Brasil (1908), Rio de Janeiro, Topbooks, 1996; Oliveira Martins, História de Portugal, Lisboa, Liv. de António Maria Pereira, 1880, v. 2 ; Jorge Pedreira et Fernando D. Costa, D. João VI. O Clemente, Mem Martins, Círculo de Leitores, 2006. 
sur la scène européenne ${ }^{26}$. En réalité, son attention stratégique se limitait aux mouvements de l'Angleterre, alliée plus redoutée qu'aimée depuis le $\mathrm{XVII}^{\mathrm{e}}$ siècle, et à ceux du gênant voisin ibérique, avec lequel les relations s'étaient améliorées depuis 1778, même si les fantômes de l'Union des Couronnes (1580-1640) n'avaient pas encore disparu. Si, en 1793, le Portugal participe à la guerre du Roussillon aux côtés de l'Espagne contre la France, après la fin de la Terreur et la signature de l'accord francoespagnol de 1795, la politique extérieure portugaise s'inquiéta soit du danger d'une invasion de son territoire décidée à Paris ou à Madrid, soit des représailles anglaises, qui auraient conduit à la perte de l'empire ${ }^{27}$. Conscient de l'importance acquise par les provinces « dénommées par le nom générique de Brésil », D. Rodrigo observe en 1797 que le Portugal, «s'il était réduit à lui-même, deviendrait, dans un très court laps de temps, une province de l'Espagne $»^{28}$. Au contraire, Antônio de Araújo de Azevedo insistait sur la neutralité avec la France comme l'objectif stratégique fondamental pour préserver le royaume, car il craignait que, face à une invasion, la solution inévitable qu'entraînerait le déménagement de la Cour vers l'Amérique finisse par déchirer la société portugaise.

Dans l'ambiance opaque de la Cour, cette dispute divisait le personnel administratif en deux groupes que l'on nomme en général le «parti anglais » et le " parti français ». Cependant, des individus appartenant aux groupes sociaux dominants, dont le pouvoir d'action se mesurait par l'accès plus ou moins direct qu'ils avaient auprès du prince, n'en étaient pas exclus ${ }^{29}$. Pour sa part, le prince João devait faire face aussi à cet autre type de pression. Depuis la chute de Pombal, la haute noblesse tentait de profiter du règne de Maria $\mathrm{I}^{\mathrm{re}}$, et plus encore de la régence de son fils, pour récupérer la place qui avait été la sienne avant 1755. D'une part, dans les cercles les plus traditionnels, on s'indignait du « despotisme ministériel », c'est-à-dire de la prise de décision par des secrétaires d'état dont les compétences propres et les mérites précis étaient présupposés, du moins en théorie, ce qui restreignait la portée des conseils et des intrigues

(26) Valentim Alexandre, Os sentidos do Império. Questão nacional e questão colonial na crise do Antigo Regime Português, Porto, Afrontamento, 1993, p. 97 sq; Manuel Amaral, Apresentação, dans A luta política em Portugal nos finais do Antigo Regime, Lisboa, Tribuna da História, 2010, v. 1, p. 9-31.

(27) Alexandre, Os sentidos do Império... op. cit.

(28) Coutinho, Textos políticos... op. cit., v. 2, p. 49 et 48.

(29) Cf. Alexandre, Os sentidos do Império... op. cit., p. 133-134, et Graça et José Sebastião da Silva Dias, Os primórdios da maçonaria em Portugal, Lisboa, Instituto de Investigação Científica, 1986, 4v. 
des nobles titrés à la Cour. Pour ce parti aristocratique, le système évoquait l'ombre du formidable marquis et faisait ressurgir des manœuvres, dont la plus grave fut la conspiration dite des gentilshommes (fidalgos), entre 1805 et 1806, qui prétendait substituer au régent sa femme, la princesse espagnole Carlota Joaquina ${ }^{30}$. Cette conspiration a échoué. D'autre part, parmi des secteurs plus éclairés, l'option se retournait vers la reprise des lois fondamentales du royaume, ce qui incluait une nouvelle valorisation des Cortès, la traditionnelle assemblée des trois états - l'Église, la noblesse et le peuple. En termes politiques, c'était dans le passé surtout qu'on recherchait la solution aux préoccupations du présent ${ }^{31}$.

De cette manière, quoique la guerre d'Espagne contre le Portugal en 1801 n'eût visé qu'à contraindre la rupture de ce dernier avec l'Angleterre, elle provoqua tout de même la perte du territoire d'Olivença et mit ainsi en évidence la vulnérabilité du royaume, renforçant le parti anglais. Deux années plus tard, pourtant, une fois consolidée sa position à l'Est de l'Europe, Bonaparte put se concentrer sur l'ennemi britannique et son fragile allié ibérique. Face aux pressions, le régent écarta un des ministres, alors que Rodrigo de Sousa Coutinho donnait sa démission. Quelque temps après, il fut remplacé par Antônio de Araújo Azevedo. Dès ce moment-là et jusqu'à la fin de 1807 , comme à son habitude, le prince João tergiversa mais il lui fallut jouer les équilibristes, acceptant les exigences françaises croissantes, sans jamais rompre les négociations avec l'Angleterre. Quand les troupes françaises traversèrent la frontière espagnole en direction de Lisbonne, sous escorte de la marine anglaise et sous les regards médusés de la population, la Cour embarqua pour le Brésil $^{32}$. On évita ainsi l'humiliation infligée à d'autres souverains, dont le roi d'Espagne, comme le souhaitait le « parti anglais », mais on n'évita pas la naissance de tensions, qui ne se manifestèrent que plus tard, comme l'avait prévu le «parti français ». De toutes façons, de l'autre côté de l'Atlantique, la construction d'un nouvel empire s'annonçait.

(30) Cf. Pedreira et Costa, D. João VI... op. cit., p. 87-88; Alexandre, Os sentidos do Império... op. cit., p. 132-133.

(31) Esteves Pereira, O pensamento político... op. cit.; Silva Dias, Os primórdios... op. cit. Cf. encore le cas de Marco Foscarini dans Franco Venturi, Utopia and Reform in the Enlightenment, Cambridge, Cambridge University Press, 1971, p. 36 surtout.

(32) Ângelo Pereira, D. João VI Príncipe e Rei, Lisboa, Empresa Nacional de Publicidade, 1953, v. 1 ; Alan K. MANChester, «A transferência da Corte Portuguesa para o Rio de Janeiro », dans Henry H. KeIth et S. F. Edwards, Conflito e continuidade na sociedade brasileira, Rio de Janeiro, Civilização Brasileira, 1970, p. 177-217. 


\section{La Cour sous les tropiques et sa colonie en Europe (1808-1820)}

Malgré les suppliques des habitants de Bahia ${ }^{33}$, la Cour choisit de s'installer à Rio de Janeiro, siège du vice-royaume depuis 1763. On s'efforça d'y recréer - par un vrai décalque selon certains, l'Almanach de Lisbonne - les principales institutions administratives centrales: les conseils du roi appelés Tribunal des Dépêches Royales (Mesa do Desembargo do Paço) et Tribunal des Affaires Religieuses (Mesa da Consciência e Ordens), ainsi que la Cour d'Appel (Casa da Suplicação), le Conseil des Finances (Conselho da Fazenda), le Conseil Militaire Supérieur et l'Intendance Générale de la Police. À cette dernière, il incombait la surveillance des idées « dangereuses », la discipline des esclaves dans les rues, mais aussi la tâche de polir les coutumes, comme c'était le cas, par exemple, de l'hygiène publique, à la charge jusqu'alors « des eaux des pluies, des rayons du soleil et de soigneux vautours $»^{34}$. Alors, malgré l'afflux d'environ 15000 personnes qui suivaient la famille royale, l'intendant dut transformer une ville coloniale de 50000 habitants en capitale digne d'accueillir une Cour. À cette fin, à partir de son centre resserré, délimité par quatre collines et situé au bord de la baie, l'occupation du terrain se déploya par les environs de Rio. Grâce à la générosité de donateurs intéressés, le prince João s'assura une magnifique résidence dans le périmètre urbain et une autre propriété dans la plus grande île de la baie, où il séjournait en alternance avec le domaine de Santa Cruz, qui avait appartenu aux Jésuites. L'ouverture des ports du Brésil aux nations amies a été accomplie immédiatement, le commerce du port de Rio, qui servait déjà d'escale aux bateaux qui assuraient la navigation avec l'Asie et l'Afrique, s'intensifia, ce qui augmenta le nombre d'esclaves et d'étrangers et contribua à créer une énorme variété de sons et de couleurs dans la ville ${ }^{35}$.

Plus important encore : la presse apportée par les voyageurs introduisit l'imprimerie dans l'Amérique portugaise. Outre la Gazette de Rio de Janeiro, l'Imprimerie Royale publia d'innombrables décisions royales, les premiers sermons, certaines Réflexions pour améliorer le climat de

(33) Instituto Histórico e Geográfico Brasileiro, boîte 102, dossier 3 (1808).

(34) João CAPISTRANo de ABREu, « Três séculos depois » (1907), dans Capitulos de história colonial, Rio de Janeiro, Civilização Brasileira / MEC, 1976, p. 189-213, p. 210. 2008 (NDLR).

(35) Pour une synthèse, voir Armelle Enders, Nouvelle Histoire du Brésil, Paris, Chandeigne, 
Rio de Janeiro et, un peu plus tard, des nouvelles et des poèmes ${ }^{36}$. Avec quelque retard, la Bibliothèque Royale traversa également l'océan et fut rendue publique. Tout aussi indispensable, on s'occupa de l'organisation de la musique et des cérémonies religieuses, qui réglaient toujours la vie de la famille royale, ainsi que de la construction d'un théâtre, activité jusqu'alors presque inconnue de la population locale mais qui faisait partie de la routine de la Cour à Lisbonne ${ }^{37}$. Cet authentique processus de civilisation fit bientôt naître des espoirs enthousiastes. En 1811, depuis le Portugal, l'évêque Azeredo Coutinho se félicitait parce que « ma patrie va jouir des prérogatives de premier empire du Nouveau Monde ». Témoin oculaire des événements, le prêtre Luís Gonçalves dos Santos écrivit plus tard, dans ses Memórias para servir à história do Brasil, que le prince João avait donné « les preuves les plus évidentes [...] qu'il était venu au Brésil pour créer un grand empire $»^{38}$. En effet, la déclaration de guerre à la France, l'activité fébrile de Sousa Coutinho, qui était revenu au cabinet royal, l'occupation de la Guyane en 1809 et, en 1811, l'intervention dans l'actuel Uruguay, en sont des preuves. Bien plus, loin de la haute noblesse, dont le plus gros effectif resta au Portugal, le régent devenait comme libéré de tous les ennuis qui l'avaient tourmenté les années précédentes. Cependant, Rio de Janeiro, devenue résidence de la Cour, comme l'a remarqué Maria Odila da Silva Dias, s'est transformée en une véritable métropole des tropiques ${ }^{39}$, c'est-à-dire en une nouvelle Lisbonne au sud de l'Équateur, capable d'attirer, comme un puissant aimant, des arrivistes de toutes sortes et venus de toutes parts. Pourtant, au même moment, la ville catalysait ainsi des tensions, non seulement vis-à-vis du royaume en Europe, dévasté par les invasions françaises et par la présence d'intérêts divergents, mais aussi par rapport aux autres capitaineries d'Amérique.

(36) Maria Beatriz Nizza da Silva, A Gazeta do Rio de Janeiro (1808-1822) : cultura e sociedade, Rio de Janeiro, EdUERJ, 2007; Ana Maria de Almeida Camargo et Rubens Borba de Moraes, Bibliografia da Impressão Régia do Rio de Janeiro, São Paulo, EdUSP / Kosmos, 1993, 2v.; Lúcia Maria Bastos P. Neves et Luiz Carlos Villalta (éds.), Quatro novelas nos tempos de D. João, Rio de Janeiro, Casa da Palavra, 2008.

(37) Cf. Maria Beatriz Nizza da SiLva, Vida privada e quotidiano no Brasil na época de D. Maria I e D. João VI, Lisboa, Estampa, 1993; Ilmar R. de Mattos, " Rio de Janeiro », dans Ronaldo Vainfas et Lúcia Bastos P. Neves (dir.), Dicionário do Brasil joanino, 1808-1821, Rio de Janeiro, Objetiva, 2008, p. 393-397.

(38) Cf. Rubens Borba de Moraes (dir.), Obras economicas de J. J. da Cunha Azeredo Coutinho (1794-1804), São Paulo, Ed. Nacional, 1966, p. 62; Luiz Gonçalves dos SANTos, Memórias para servir o Reino do Brasil (1825), Belo Horizonte/ São Paulo, Itatiaia/ EDUSP, 1981, v. 1, p. 187.

(39) Maria Odila da Silva Dias, «A interiorização da metrópole (1808-1853) » dans $A$ interiorização da metrópole e outros estudos, São Paulo, Alameda, 2005, p. 7-37. 
En effet, au Portugal, cette situation ne provoquait que tristesse et ressentiments. Avec le départ de la famille royale, le gouvernement échut à un Conseil de Régence, soucieux de maintenir de bonnes relations avec Napoléon selon les instructions du prince-régent ${ }^{40}$. Cependant, en fonction de la forte dimension symbolique que revêtait l'absence du souverain, il se créa un sentiment d'abandon collectif, les Portugais se sentant un peu comme des orphelins, parce que le système monarchique restait toujours fondé sur le principe de la grande famille dans laquelle la figure du roi, indissociablement unie à celle de la nation, remplaçait le père ${ }^{41}$. Bien que quelques sujets aient louangé la décision du régent, la majorité des Portugais ressentit le transfert de la Cour vers Rio de Janeiro comme une "fuite tumultueuse », un acte de lâcheté, donnant "l'impression d'abandonner la maison qui brûle », tandis que la population de Lisbonne, se trouvant «abandonnée de cette façon entre les mains des Français », exprimait des «propos blasphématoires et de désespoir $»^{42}$. Néanmoins, des couches de la société portugaise ne manquèrent pas l'occasion de souhaiter la bienvenue aux occupants. Quelques lettrés attendaient avec impatience les changements politiques que l'instauration prévisible du Code Napoléonien promettait; les élites marchandes prévoyaient de nouveaux marchés à ouvrir; la haute noblesse enfin devint un des piliers du gouvernement de Junot dans l'espoir de récupérer l'autonomie et le prestige d'antan. Pour toutes ces raisons, en mai 1808, plusieurs membres de ces différentes élites envoyèrent une députation à Bayonne dans le but de solliciter de Napoléon la désignation d'un nouveau roi ${ }^{43}$.

Les réactions cependant ne tardèrent pas. Vers la mi-décembre 1807, la population insultait déjà les soldats français à Lisbonne, en criant : «Vive le Portugal, vive les Cinq Plaies et mort à la France ! $»^{44}$. Le

(40) José Acúrsio das Neves, História geral da invasão dos franceses em Portugal e da restauração deste reino (1810), Porto, Afrontamento, s/d, v. 1, p. 220.

(41) Id., p. 223. Pour l'Espagne et ses possessions, voir François-Xavier GuERRA, Modernidad e independencias. Ensayos sobre las revoluciones hispanicas, México, Mapfre / Fondo de Cultura Ecónomica, 1993, p. 150-156.

(42) Citations de José Liberato Freire de Carvalho, Ensaio histórico-politico sobre a Constituição e Governo do Reino de Portugal (1830), Lisboa, Imprensa Nevesiana, 1843, p. 197, et de O Portuguez (1814) dans Georges BoIsvert, Un pionnier de la propagande libérale au Portugal : João Bernardo da Rocha Loureiro (1778-1853), Paris, Fundação Calouste Gulbenkian / Centre Culturel Portugais, 1982, p. 70.

(43) Lúcia Bastos P. Neves, Napoleão Bonaparte. Imaginário e política em Portugal c. 1808-1810, São Paulo, Alameda, 2008 p. 98-100.

(44) Id., p. 95. La référence aux "Cinq Plaies" fait allusion au Miracle d'Ourique, un des mythes fondateurs de la monarchie portugaise, selon lequel le Christ serait apparu, à celui qui devint plus tard le premier roi du Portugal, à l'occasion d'une bataille décisive en 1139 . 
$1^{\text {er }}$ février, le Conseil de Régence fut abrogé et la maison de Bragance destituée; d'autres mesures semblables, administratives et fiscales, suivirent et drainèrent une partie du numéraire en circulation. Des pamphlets, comme ces vers intitulés Protection à la française, dénonçant celle-ci comme ne visant qu'à « sauver les biens, extorquer de l'argent [et ne] laisser derrière soi que de la pauvreté », commencent à circuler ${ }^{45}$. En mai 1808, sans pouvoir apporter une aide effective au royaume, la Cour envoie en Europe une note destinée à éclairer ses positions envers la France, mais qui, de ce fait, libère les Portugais pour faire la guerre aux soldats de Napoléon ${ }^{46}$. Entre-temps, en mars, le roi et le prince héritier d'Espagne furent obligés d'abdiquer, ce qui causa une révolte populaire dans le pays deux mois plus tard. D'où, en juin, à Porto, l'irruption de la résistance portugaise avec l'appui des troupes d'occupation espagnoles. Décidé à Londres de manière indépendante, le débarquement au Portugal de soldats anglais en août 1808, rendit la situation intenable pour les occupants français, dont la retraite fut décidée par la convention de Sintra, signée entre la France et l'Angleterre à la fin du mois. Une véritable amnistie était consentie aux collaborateurs de Junot, créant ainsi de nouveaux ressentiments. La deuxième invasion française, de mars à mai 1809, sous le commandement de Soult, et la troisième, d'août à septembre 1810, sous celui de Masséna, n'ont pas duré longtemps, mais toutes ensembles, elles furent suffisantes pour dévaster le pays et engendrer des conséquences calamiteuses pour les années qui suivirent ${ }^{47}$. De l'autre côté de l'océan, l'ouverture des ports américains en 1808 et la signature des traités commerciaux avec l'Angleterre en février 1810 , contribuèrent à affaiblir les structures économiques du Portugal ${ }^{48}$.

Face à la crise, les couches de la population les plus modestes et même les catégories urbaines moyennes eurent recours au surnaturel, à l'idée d'un sauveur, dans la perspective millénariste du sébastianisme, cette croyance élaborée après la disparition du roi Sébastien dans la bataille de Ksar-el-Kébir en $1578^{49}$. Quant aux groupes dominants, le rétablissement

(45) Réimprimé à Rio de Janeiro, Impressão Régia, 1809, p. 4, adaptation.

(46) Manifesto ou exposição fundada, e justificativa da corte de Portugal a respeito da França, Rio de Janeiro, Impressão Régia, 1808.

(47) Lúcia Bastos P. Neves, Napoleão... op. cit., p. 102-111.

(48) Cf. Jorge Borges de MACEDo, O Bloqueio Continental. Economia e guerra peninsular, Lisboa, Gradiva, 1990; Valentim AleXandre, Os sentidos do império... op. cit.; Jorge Miguel VianA Pedreira, Estrutura industrial e mercado colonial : Portugal e Brasil (1780-1830), Lisboa, Difel, 1994.

(49) Lúcia Bastos P. Neves, Napoleão... op. cit., p. 259-264; Jacqueline Hermann, No reino do desejado : a construção do sebastianismo em Portugal (séculos XVI e XVII), São Paulo, Companhia das Letras, 1998; Lucette VAlensi, Fábulas da memória : a batalha de Alcácer Quibir e o mito do sebastianismo, Rio de Janeiro, Nova Fronteira, 1994. 
de la paix en Europe les laissa perplexes et mécontents de la présence durable du prince João en Amérique, parce qu'ils voyaient le royaume du Portugal réduit «à l'humiliante qualité de colonie $»^{50}$. Alors, en mars 1816, la mort de la reine et le début du nouveau règne raniment l'espoir d'un retour de la Cour au Portugal. Mais le deuil sembla avoir bouché les oreilles de la Couronne, qui ne sort de sa léthargie que pour déterminer une nouvelle campagne d'annexion de l'Uruguay. Signaux de l'accumulation des tensions, la révolte du Pernambouc éclata en mars 1817 et, trois mois plus tard, la conspiration de Gomes Freire, général prestigieux qui avait lutté dans la Légion Portugaise, un corps de la Grande Armée, fut réprimée au Portugal. En réalité, l'inactivité du roi, qui tergiversait toujours, avait une origine bien précise. À cette époque, après la mort de Rodrigo de Sousa Coutinho en 1812, Antônio de Araújo de Azevedo était revenu au cabinet et conduisait des démarches afin de promouvoir un rapprochement avec la France, en éloignant ainsi la couronne portugaise de la tutelle anglaise. Ces efforts attirèrent à Rio le duc du Luxembourg, pour qui il était clair que la mort de Maria $\mathrm{I}^{\mathrm{re}} \mathrm{n}$ 'impliquait aucun changement politique, car son fils gouvernait déjà depuis longtemps. Toutefois, selon ce représentant français, une vive polémique s'était établie parmi le corps diplomatique sur le lieu du « couronnement » du nouveau souverain, puisque ce choix impliquait l'établissement définitif du siège de la monarchie ${ }^{51}$. Pour cette raison, le Portugal insistait pour qu'un acte d'une telle importance ne puisse être célébré sans la présence des Cortès ${ }^{52}$. Cependant, au grand dam des Portugais, ce fut Rio de Janeiro qui assista avec émerveillement à la cérémonie d'acclamation de João VI comme roi du Portugal, du Brésil et des Algarves au début de l'année suivante. Pareille cérémonie était inédite en Amérique; pour en assurer la magnificence et en faire ressortir la dimension fortement symbolique, on fit appel à la contribution - pour utiliser l'expression de Lilia Schwarcz - de la colonie Lebreton, formée d'artistes et d'artisans français récemment arrivés en ville. Grâce à l'imposante architecture éphémère qu'ils imaginèrent, le Largo do Paço, l'espace

(50) Silvestre Pinheiro Ferreira, « Memórias Políticas sobre os abusos gerais e modo de os reformar e prevenir a revolução popular redigidas por ordem do príncipe Regente », RIHGB, $\mathrm{n}^{\circ} 47$, 1884 , p. 2.

(51) Les souverains portugais n'étaient pas couronnés, mais seulement acclamés.

(52) Rapport de l'Ambassade du Duc de Luxembourg (1816), dans Jean de Pins, Sentiment et diplomatie d'après des correspondances franco-portugaises. Contribution à l'histoire des mentalités au début du XIXe siècle, Paris, Fond. Calouste Gulbenkian, 1984, p. 538-539. 
autour du palais, se vit transformé en véritable place impériale ${ }^{53}$. L'objectif était clair. Il s'agissait de renforcer l'image du roi, en donnant à la cérémonie d'acclamation la dimension d'un événement capable d'assurer autant le poids politique du Brésil dans l'empire portugais que la suprématie de Rio sur les autres provinces. Dans la même perspective, le souverain commanda au courtisan José da Silva Lisboa un Mémoire qui, en rappelant les principaux bénéfices politiques que le nouveau monarque avait apportés jusqu’à sa « fastueuse Acclamation », inscrivît « une époque aussi grandiose dans les annales de l'Amérique ${ }^{54}$.

Cependant, cet investissement n'eut pas les mêmes répercussions de l'autre côté de l'Atlantique. La rancœur des Portugais se traduisit par le surnom de « gouvernement Tupinambá ${ }^{55}$ » attribué à l'administration au Brésil. En 1819, le journal O Campeão Português, Amigo do Rei e do Povo affirmait que le Portugal se sentait condamné « à une décadence progressive » et obligé à de constants sacrifices. Les Portugais se voyaient donc « sans roi et quasiment sans patrie », gémissant en silence et toujours « orphelins ». Et il concluait : « sans peuple, il n'y a pas de trône ni de couronne, mais il peut y avoir, et il y a déjà eu, des peuples sans trône ni couronne $[\ldots]{ }^{56}$. Les conditions étaient réunies pour ce qu'on appela la Régénération d'août 1820, inspirée de l'exemple récent de l'Espagne et dotée d'un caractère libéral. Ce soulèvement exigeait alors la convocation des Cortès pour l'élaboration d'une constitution, le retour du souverain en Europe et le rétablissement de la place que le Portugal estimait mériter au sein de l'empire.

\section{Le Brésil et l'empire (1820-1822)}

Les échos de la rébellion portugaise parvinrent en Amérique au début de l'année 1821, d'abord au Pará et à Bahia, puis à Rio de Janeiro, qui comptait à l'époque plus de 110000 habitants. Dès lors, les conceptions du Vintismo [le mouvement de l'année vinte, vingt] commencèrent à se répandre grâce à une surprenante quantité de journaux et de pamphlets

(53) Cf. Lilia M. Schwarcz, O Sol do Brasil. Nicolas-Antoine Taunay e as desventuras dos artistas franceses na corte de D. João, São Paulo, Companhia das Letras, 2008.

(54) Memória dos beneficios políticos do governo de el-rey nosso senhor D. João VI [...], Rio de Janeiro, Impressão Régia, 1818 et Gonçalves dos SAntos, Memórias..., v. 2, p. 151.

(55) À la période coloniale, c'était la dénomination la plus générale des peuples indiens qui occupaient le littoral. Cf. Ronald RAminelLI, « Tupinambá », dans Ronaldo VAINFAS (dir.), Dicionário do Brasil colonial, Rio de Janeiro, Objetiva, 2000, p. 566.

(56) Londres, v. 1, $\mathrm{n}^{\circ} 1$. 
politiques. Certains venaient de Lisbonne, d'autres étaient imprimés à Rio et à Bahia, et plusieurs atteignirent le Maragnon, le Pernambouc, São Paulo, le Minas Gerais et même l'actuel Uruguay. Ces ouvrages de circonstance critiquaient presque toujours le gouvernement absolu et cherchaient à diffuser les principes du constitutionalisme monarchique de manière le plus souvent accordée à la culture orale alors dominante, sous forme de lettres ou de dialogues - quand ils ne parodiaient pas tout à fait des œuvres et des prières religieuses, comme les catéchismes constitutionnels, les « Notre Père constitutionnels » et les « Je vous salue Marie constitutionnels ». En effet, normalement limitées au cercle de l'élite intellectuelle pour laquelle les boutiques d'apothicaire, les cafés, les librairies et les loges maçonniques formaient de nouveaux espaces de sociabilité, en peu de temps les discussions atteignirent les rues et rassemblèrent différents acteurs ${ }^{57}$. Aux carrefours de Bahia, par exemple, les murs exhibèrent des affichettes manuscrites qui, malgré des critiques vexantes adressées contre le « despotisme ministériel », restaient fidèles à l'idée de l'unité des domaines portugais : « Héros bahianais! Aux armes! La gloire vous interpelle! Vos illustres aïeux du Douro et du Tage vous ont montré l'exemple et vous attendent. Criez avec audace : Vive la Constitution du Brésil et le roi qui ne la refusera pas! $»^{58}$.

Les débats apparemment prenaient leur envol. Subitement, l'Amérique portugaise semblait concourir à la création d'un nouvel espace public de la politique. Néanmoins, s'il existait d'un bord à l'autre de l'Atlantique une quasi-unanimité critique envers les corcundas (les partisans de l'Ancien Régime), les publications de 1821 ne remettaient pas encore en question l'unité de l'empire. En réalité, en accueillant le siège de la monarchie portugaise en 1808, le Brésil avait, de ce fait, cessé d'être une colonie, ayant obtenu, dès 1815 , en outre, le statut de royaume, uni

(57) Lúcia Bastos P. Neves, Corcundas e Constitucionais : a cultura política da Independência (1820-1823), Rio de Janeiro, Revan / Faperj, 2003, p. 39-44. Voir aussi FrançoisXavier Guerra, Annick Lempèriere et al., Los espacios públicos em Iberoamérica. Ambiguedades y problemas. Siglos XVIII-XIX, Mexico, FCE, 1998; Dena Goodman, « Public Sphere and Private Life : toward a synthesis of current historiographical approaches to the Old Regime », History and Theory, Middletown, 31 (1) :1-20, 1992; Roger CHARTIER, Espacio público, crítica y desacralización en el siglo XVIII. Los origenes de la Révolución francesa, Barcelona, Gedisa, 1995; Jurgen HaBERMAS, L'espace public. Archéologie de la publicité comme dimension constitutive de la société bourgeoise, Paris, Payot, 1993 ; A. H. de OliveIra Marques, História da maçonaria em Portugal : das origens ao triunfo, Lisboa, Presença, 1989; Marco Morel et Françoise Jean de O. SouZA, O poder da Maçonaria, Rio de Janeiro, Nova Fronteira, 2005.

(58) Arquivo Histórico do Itamaraty, Rio de Janeiro, Coleções Especiais, capitania da Bahia, boîte 195 , paquet 01 , dossier 07 . C'est nous qui soulignons. 
au Portugal et à l'Algarve. Dans un premier temps, le roi hésita à revenir. Bien que cette option risquât de légitimer la « révolution », le conseil du comte de Palmela, venu en urgence de Lisbonne, fut le retour de la Cour à l'ancienne capitale afin d'endiguer le mouvement. D'autre part, malgré la menace de la perte du trône des Bragance en Europe, Vilanova Portugal, le principal ministre, insistait sur le fait que le roi devait rester en Amérique, de manière à éviter la contagion des idées les plus radicales au Brésil et à y préserver ainsi l'ordre traditionnel. Le 26 février 1821, les troupes portugaises à Rio de Janeiro entreprennent un mouvement qui exige du souverain la démission des membres du gouvernement, le serment immédiat des bases de la future Constitution et, en attendant l'élaboration de celle portugaise par les Cortès, l'adoption de la Charte espagnole. L'épisode pourtant fut rapidement déjoué par le prince Pedro, l'héritier de la couronne. Fatigué, peut-être même malade, le roi se décide enfin à partir et détermine l'élection des députés brésiliens pour les Cortès de Lisbonne, en conformité avec ce qu'établissait la Constitution de Cádiz, dont un décret du 21 avril instituait l'entrée en vigueur provisoire en Amérique ${ }^{59}$. Cependant, à la suite de troubles sur la place du Commerce à Rio, la décision fut annulée le jour même. Moins d'une semaine plus tard, João VI embarquait, laissant son fils comme régent au Brésil ${ }^{60}$.

Âgé de 22 ans à peine et sans aucune sympathie pour les Cortès, le jeune prince chercha l'appui du secteur de l'élite brésilienne composée en grande partie par des hommes qui étaient passés par l'Université de Coimbra, qui se souciaient de l'unité de l'empire et qui avaient l'expérience de l'administration. C'est l'élite dite coimbrä $\tilde{a}^{61}$. En outre, dans un pays où le tiers de la population au moins était constitué d'esclaves, l'exemple de Saint Domingue faisait craindre des émeutes et plaçait le maintien de l'ordre au-dessus de tout ${ }^{62}$. Par contre, au long du second semestre 1821 , les nouvelles des débats aux Cortès laissaient transparaître de plus en plus

(59) Au sujet de la santé du roi, cf. Bilhetes a Tomás Antônio Vilanova Portugal (1816-1821), Rio de Janeiro, Biblioteca Nacional, Divisão de Manuscritos, 5, 1, 40.

(60) Maria Beatriz Nizza da Silva, "Liberalismo e separatismo no Brasil : 1821-1823", Lisboa, Centro de História da Cultura da Universidade Nova de Lisboa, 1986 (Separata); Valentim Alexandre, Os sentidos do império... op. cit., p. 529-539; Lúcia Bastos P. Neves, Corcundas e Constitucionais... op. cit., p. 249-256.

(61) Voir Roderick J. Barman, Brazil : The Forging of a Nation (1798-1852), Standford, University Press, 1988, p. 65 sq.

(62) Au contraire de plusieurs auteurs, ce texte-ci ne considère pas l'esclavage comme responsable de la forme que prit le processus d'indépendance au Brésil, mais le considère plutôt comme le résultat même de l'ambiance sociale et culturelle luso-brésilienne, où l'égalité ne découlait pas encore d'un droit humain fondamental et inaliénable, mais exclusivement de la lettre de la loi. 
clairement les objectifs de la Régénération portugaise : soumettre le roi, qui avait abandonné ses sujets, à l'autorité de l'assemblée et rétablir la suprématie lusitanienne sur le reste de l'empire. Face aux décrets du 29 septembre qui exigeaient le retour du régent au Portugal, le prince prit la décision inverse de celle de son père. Il préféra rester, avec l'idée probable d'instaurer au Brésil une monarchie éclairée, certes, mais encore absolue. Annoncée le 9 janvier 1822, le jour du Fico (c'est-à-dire, je reste), cette intention se manifesta en réponse à une pétition qui avait recueilli 8000 signatures, mais fit que les troupes portugaises essayèrent d'obliger sans succès le régent à embarquer. 1822 ne sera plus l'année du « constitutionnalisme », comme l'avait été 1821 , mais bien celle du « séparatisme $»^{63}$.

Dans les six mois qui suivirent, les mesures prises par les Cortès - comme la décision d'abolir des institutions fondées à l'arrivée du prince João au Brésil - renforcèrent l'union des élites locales et aggravèrent le climat d'animosité contre les Européens, tandis que les décisions du prince Pedro en Amérique semblaient aux yeux des députés de Lisbonne une manifestation de ses tendances anticonstitutionnelles. Comme on peut le lire dans une lettre du sacristain d'un lieu nommé Tambi et adressée à un étudiant de Rio (Carta do sacristão de Tambi ao estudante constitucional do $R_{i o}{ }^{64}$ ), il se créa ainsi une guerre «plus de plume que de langue ou d'épée », qui fréquemment devenait incompréhensible à cause du décalage dans l'échange des nouvelles de part et d'autre de l'Atlantique ${ }^{65}$. D'un côté, les brasilienses ou brasileiros lisaient dans les écrits imprimés au Portugal la volonté de provoquer la désunion du Brésil, par les insultes qu'ils contenaient. De l'autre, en raison de la menace à l'intégrité de l'empire que leur attitude semblait représenter, le Portugal soulignait l'ingratitude des Américains envers la mère-patrie, qui leur avait concédé tant de profits grâce au constitutionalisme ${ }^{66}$. C'est dans cette ambiance que les événements se précipitèrent.

Une semaine après le Fico, le régent nomme à la tête d'un ministère José Bonifácio de Andrade e Silva, un remarquable coimbrão, âgé de 59 ans et ami proche de Sousa Coutinho. Un mois plus tard, un Conseil de Procureurs des provinces fait l'effort de resserrer les liens du gouver-

(63) Manuel de Oliveira Lima, O movimento da independência: 1821-1822, Belo Horizonte / São Paulo, Itatiaia / Edusp, 1989.

(64) Cette lettre, imprimée dans un périodique de Rio le 8 janvier 1822, circule par la suite comme un pamphlet indépendant.

(65) Revérbero Constitucional Fluminense, Rio de Janeiro, nº 9, 8 janvier 1822.

(66) Lúcia Bastos P. Neves, Corcundas e Constitucionais..., op. cit., p. 313-342. 
nement de Rio de Janeiro avec les autres régions. Le 30 avril, le journal Revérbero Constitucional Fluminense, sous la direction de Gonçalves Ledo, chef des brasilienses, autre faction des élites, moins soucieuse de l'ensemble de l'empire que de ses intérêts locaux, dénonce les Cortès comme incapables de poursuivre un dialogue et propose l'indépendance du Brésil. Le 23 mai 1822, le Portugais José Clemente Pereira, président de la Chambre municipale de $\mathrm{Rio}^{67}$, apporte au prince la demande de convocation d'une Assemblée brasílica ${ }^{68}$. Au même moment, le congrès à Lisbonne discutait encore un projet sur les relations commerciales entre le Brésil et le Portugal. Il avait pour but de concilier les intérêts productifs et commerciaux de l'ancienne métropole avec ceux de l'ex-colonie, faisant du royaume uni un marché unique, fortement intégré et protégé de l'extérieur. Par conséquent, bien que l'idée d'indépendance cheminât pas à pas, aucun des témoignages de l'époque ne semble indiquer l'intention des Cortès de rétablir l'exclusif commercial comme facteur décisif du séparatisme qui s'affirmait, au contraire de ce que l'historiographie a l'habitude d'affirmer. Curieusement, de ces supposés mouvements antérieurs de contestation depuis 1'Inconfidência de Minas Gerais de 1789, la seule évocation fut celle de la Révolution du Pernambouc en 1817 par frère Caneca, mais avec l'objectif plutôt d'éviter l'exaspération des conflits entre les Portugais d'Europe et ceux d'Amérique ${ }^{69}$.

À Rio de Janeiro, non plus, le prince n'avait pas pour intention de rompre les liens qui rattachaient le Brésil au Portugal, bien qu'il cherchât à assurer une autonomie que les Cortès lui refusaient. Il lui fallait réaffirmer un pouvoir local centralisé, capable d'éviter le démantèlement de ces « cinq groupes ethnographiques, liés par la communauté active de la langue et passive de la religion, modelés par les conditions de cinq différentes régions », dont les habitants, pourtant, ne s'estimaient pas « entre eux de façon particulière $\gg{ }^{70}$. Dans un certain sens, une fois décidée la convocation de l'Assemblée brasílica le 3 juin, l'indépendance était consommée. Le mois suivant, le congrès de Lisbonne abandonnait la discussion sur le projet des relations commerciales pour ne plus jamais y revenir. Le 14 juillet, le prince Pedro envoyait des troupes vers Bahia, qui avait déclaré fidélité aux propo-

(67) C'est-à-dire le maire de la ville.

(68) L'adjectif n'est pas encore défini à l'époque : on peut trouver : brasileiro, brasiliense et brasílico.

(69) Voir « Dissertação sobre o que se deve entender por pátria do cidadão e deveres deste para com a mesma pátria », dans Evaldo Cabral de Mello (éd.), Frei Joaquim do Amor Divino Caneca, São Paulo, Ed. 34, 2001, p. 53-99.

(70) João Capistrano de Abreu, «Três séculos depois »..., op. cit., p. 213. 
sitions libérales des Cortès de Lisbonne. Le $1^{\text {er }}$ août, un décret du prince déclare considérer tous les militaires portugais qui débarqueraient sans son consentement comme des ennemis. Pour justifier cette mesure, il allègue que le congrès européen persistait, « ayant recours à la force si nécessaire, dans le même système erroné et évidemment injuste, de recoloniser le Brésil, malgré le fait que celui-ci avait déjà proclamé son indépendance politique ». Néanmoins, il assurait aussi le maintien des relations commerciales amicales entre les deux royaumes, « pour sauvegarder l'union politique, que je tiens beaucoup à maintenir ». L'apparente contradiction peut être expliquée. À ce moment-là, "indépendance" signifiait "autonomie" et ne conduisait pas forcément à la séparation avec le Portugal, surtout quand cette indépendance avait été déclarée par l'héritier de la couronne lusitaine lui-même ${ }^{71}$. À la même date, le Manifeste aux Peuples du Brésil, écrit par Gonçalves Ledo, et, le 6 août, le Manifeste aux Nations Amies, rédigé par José Bonifácio, présupposaient la séparation ${ }^{72}$. Si tous deux attribuaient au despotisme des Cortès la responsabilité des événements, le premier considérait la situation comme irréversible, tandis que le second hésitait encore à renoncer au projet d'un empire unique. Dans cette optique, il n'est pas surprenant que le célèbre Cri de l'Ipiranga du 7 septembre 1822, proclamé, ou non, par le prince Pedro sur les rivages d'un ruisseau à São Paulo - actuellement la date officielle de l'indépendance -, ait eu si peu de répercussions parmi les contemporains et que c'est le 3 juin qui fut d'abord considéré comme « le jour anniversaire de la [...] Régénération politique » du Brésil, le jour « où fut rompue la chaîne de la dépendance servile et coloniale; $[\ldots]$ où le cancer fut arraché du corps gigantesque $»^{73}$.

$*$

Moment fondateur de tous les pays américains à l'exception du Canada, l'indépendance est restée, jusque très récemment, pour l'historio-

(71) BRASIL, Decreto declarando inimigas as tropas mandadas de Portugal sem consentimento de S. A. R. e dando providências para o caso de violências, (Rio de Janeiro), Imp. Nacional, (1822), f. 1 et Lúcia Bastos P. Neves et Guilherme P. Neves, «Independência - Brasil », article pour le $2^{\mathrm{e}}$ volume, en préparation, de Javier FERNÁNDEZ SEBASTIÁN (dir.), Diccionario político y social del mundo iberoamericano : la era de las revoluciones, 1750-1850, Madrid, Fund. Carolina / Sociedad Estatal de Conmemoraciones Culturales / Centro de Estudios Políticos y Constitucionales, 2009.

(72) Manifesto de S. A. R. o Príncipe Regente Constitucional e defensor perpétuo do reino do Brasil aos povos deste reino, Rio de Janeiro, Imp. Nacional, 1822; Manifesto do Príncipe Regente do Brasil aos governos e nações amigas, Rio de Janeiro, Imp. Nacional, 1822.

(73) O Macaco Brasileiro, Rio de Janeiro, n 2, 1822; Lúcia Bastos P. Neves, Corcundas e Constitucionais..., op. cit., p. 370. 
graphie brésilienne, le point final d'un processus continu et linéaire qui, tout au long du XVIII ${ }^{\mathrm{e}}$ siècle et des premières décennies du XIX ${ }^{\mathrm{e}}$, forgea une conscience nationale et mit fin aux traditions coloniales ${ }^{74}$. Sans doute, durant cette période que s'étend de la montée du marquis de Pombal au pouvoir jusqu'au retour de João VI en Europe, le rôle de l'Amérique et de ses habitants devint décisif. Néanmoins, dans la perspective adoptée ici, l'évènement de 1822 ne représenta pas une rupture, mais plutôt une dispute pour l'hégémonie à l'intérieur d'un vaste empire composite ${ }^{75}$, ayant recours aux valeurs des Lumières portugaises et en accord avec les dynamismes qui découlèrent des conflits entre les élites de l'un et l'autre côté de l'Atlantique.

Il y a longtemps, cependant, à propos de l'Europe du XVIII ${ }^{\mathrm{e}}$ siècle, Franco Venturi faisait remarquer que l' « un des problèmes les plus difficiles est de comprendre au juste jusqu'à quel point cette rationalisation est technique, c'est-à-dire toute instrumentale, à l'intérieur de structures mentales que la dominent et l'encadrent - et jusqu'à quel point au contraire cette mentalité nouvelle est capable d'éroder et de dissoudre les cadres eux-mêmes que la maintiennent enchaînée et l'entravent ${ }^{76}$. Or, dans le monde luso-brésilien, plusieurs indices suggèrent que la première option prévalut, assurant continuité des valeurs traditionnelles c'est-à-dire religieuses. L'évêque Azeredo Coutinho, fondateur du séminaire d'Olinda, institution modelée sur l'Université de Coimbra par laquelle on prétendait enraciner les propositions de D. Rodrigo au Nouveau Monde, estimait, aux environs de 1800, non seulement que les étudiants ne seraient de « bons citoyens » que dans la mesure où ils seraient de « bons chrétiens », mais il comptait aussi sur « le prêtre de hautes terres ${ }^{77}$ et des broussailles, sage et instruit en sciences naturelles », pour devenir l'instrument « propice à la grande entreprise des découvertes de la nature et de ses trésors $\gg^{78}$. En 1822, un air entonné dans les rues de Lisbonne surprit le comte Pecchio, un milanais libéral qui fuyait la répression en son propre pays :

(74) À titre d'exemple, voir José Honório RodRIgues, A independência : revolução e contrarevolução, Rio de Janeiro, Francisco Alves, 1975-1976.

(75) John H. Elliott, «A Europe of Composite Monarchies », Past \& Present, n 137, Nov. 1992, p. 48-71.

(76) Franco Venturi, "Les lumières dans l'Europe du $18^{\mathrm{e}}$ siècle », dans Europe des lumières : recherches sur le $18^{e}$ siècle, Paris, Mouton, 1971, p. 4-34, p. 14

(77) En portugais, sertões - mot qui désigne l'intérieur par opposition au littoral. La traduction française de Os sertões (1902) de Euclides da Cunha eut pour titre Hautes terres.

(78) José Joaquim da Cunha de Azeredo Coutinho, « Estatutos do seminário episcopal de N. Senhora da Graça da cidade de Olinda » (1798), dans Severino Leite NogueIRA, O seminário de Olinda e seu fundador, Recife, Fundarpe, 1985, p. 343 ; Borba de MoraEs (dir.), Obras econômicas..., op. cit., p. 212. 
Já pouco tarda o momento

Da nossa consolação

Em que há de baixar dos Céus

A nossa Constituição.

Après tout - c'était la question qu'il se posait - « une loi rédigée par une Chambre de représentants du peuple n'aurait-elle par elle-même un caractère assez auguste et vénérable ? ${ }^{79}$. Ces deux exemples illustrent à merveille combien la religion restait au Portugal et au Brésil cette « organisation du monde humain-social constituant l'ordre qui tient les hommes ensemble comme un ordre extérieur, antérieur et supérieur à leur volonté », comme l'écrit Marcel Gauchet. Et comme hétéronomie, la religion offrait bien des obstacles pour que surgisse l'autonomie que présuppose la démocratie, ce « pouvoir des hommes » qui prend la place de celui de Dieu, et qu'exige la pensée historique moderne ${ }^{80}$.

Par la suite, les destins parallèles des deux pays semblent confirmer cette perspective. Approuvée par les Cortès en septembre et par le serment du roi - mais non de la reine - en octobre, la Constitution portugaise de 1822 fut suspendue le 3 juin 1823 par le mouvement dit de Vila Francada avec la promesse d'une nouvelle Charte qui ne se matérialisa pas tant que le souverain vécut. Au Brésil, si Pedro Ir fut proclamé empereur sur la place publique, à l'occasion d'une grande fête populaire le 12 octobre 1822, après une manœuvre de l'élite coimbrã contre les membres de l'élite brasiliense, on le couronna le $1^{\text {er }}$ décembre suivant, sous l'inspiration du sacre de Napoléon. Réunie le 3 mai 1823, l'assemblée constituante est dissoute par les troupes le 12 novembre. Le jour suivant, l'empereur ordonne la rédaction d'une nouvelle Constitution et l'octroie le 25 mars 1824. Après la mort de son père, il la fait adapter pour le Portugal en 1826, où elle devint une pomme de discorde qui conduisit à une longue guerre civile. En Amérique, les provinces qui restèrent fidèles aux Cortès - Pará, Maragnon et Bahia - furent assujetties, tandis que l'assemblée ordinaire, qui se rassembla en 1826, conteste de plus en plus le pouvoir de Pedro ${ }^{\text {er }}$. En abdiquant en 1831, celui-ci lègue au nouveau pays une décennie de conflits, qui ne seront apaisés qu'à partir de la montée sur le trône de son

(79) José Pecchio, Cartas de Lisboa : 1822, Lisboa, Livros Horizonte, 1990, p. 21-2. Les vers disent : Bientôt viendra le moment / de notre consolation / où descendra des cieux / notre Constitution.

(80) Marcel Gauchet, Un monde désenchanté? Paris, Les Éditions de l'Atelier / Éditions Ouvrières, 2004, p. 183; et Reinhart Koselleck, Le futur passé, Paris, Éditions de l'EHESS, 1979. 
fils, Pedro II, en $1840^{81}$. En effet, comme Colmenares s'en est aperçu, les racines du passé ne cessèrent jamais de germer dans le présent.

\section{Lúcia Maria Bastos Pereira das Neves} Université de l'État de Rio de Janeiro

Chercheuse du CNPq

Bénéficiaire d'une bourse « Cientista do Nosso Estado » de la

FAPERJ

Coordinatrice principale du Projet PRONEX dirigé par José

Murilo de Carvalho

Guilherme PEREIRA DAs NEVES

Université Fédérale Fluminense

Chercheur du CNPq

Chercheur du Projet PRONEX dirigé par Ronaldo Vainfas neves.gp@gmail.com

Traduction : Diego Fonseca dos Santos dgfonseca@gmail.com 